\title{
Study on a Quantitative Analysis Method for Pulse Compression Results
}

\author{
MA Linzhi ${ }^{1, *}$, Wang Min ${ }^{1}$, WENG Chenxiang ${ }^{1}$, and SHI Zhouyan ${ }^{1}$ \\ ${ }^{1}$ Air Force Early Warning Academy, Wuhan 430019, China
}

\begin{abstract}
In order to further improve the ability of detecting and evaluating the detection performance and interference effect of the integrated signal, a quantitative analysis method (K-means quantization) for pulse compression is proposed. The method is based on the amplitude analysis of the pulse compression results. By using the improved K-means clustering method, the recognition degree of the selected target points is obtained by induction and analysis. The simulation results show that the analysis of the detection performance or interference performance of the integrated signal is consistent with the traditional analysis conclusion by using K-means quantization method. It has better measurability, objectivity and independence, which can be used for automatic evaluation of the system.
\end{abstract}

\section{INTRODUCTION}

In the detection performance analysis of integrated signal, the quantitative parameters such as radar detection are commonly used as evaluation indexes. It plays an important role in evaluating the performance of integrated signal, and has the advantages of strong objectivity and high distinction. In the process of interference analysis, especially the related interference, the pulse compression diagram is often used for corresponding analysis and evaluation, which has the advantages of wide applicability and intuitive results. In view of the deception jamming effect of the pulse compression results, the quantitative analysis method of the peak to side lobe ratio (PSLR) ${ }^{[1]}$ can achieve better results, but the ability of the coverage jamming effect is insufficient; the peak to average ratio $(\mathrm{PAR})^{[3]}$ is adopted for the coverage interference effect of the pulse compression results, but the analysis ability of deceptive jamming effect is insufficient; the subjective analysis ${ }^{[2,6]}$ or subjective analysis with parameters ${ }^{[5,7]}$ is more direct, but it is not conducive to the application of the evaluation system or to the comparative analysis, and the degree of subjective influence is heavier.

In order to synthesize the advantages of these methods, to improve the detection performance of the integrated signal and the ability to evaluate and analyze the interference effect, and to achieve the purpose of the performance of the automatic discriminant system, a method of quantitative analysis of the results of pulse compression (K-means quantization) is proposed. The quantitative parameters is obtained with the amplitude information of the pulse compression results, and it is used to evaluate the detection effect and interference effect, so as to improve the applicability and accuracy of the evaluation index ${ }^{[4]}$. And the feasibility of the quantitative analysis method in signal performance analysis and jamming effect analysis is simulated.

\section{PULSE TECHNOLOGY}

COMPRESSION

Usually, pulse compression technology is used for special radar signals, and the Linear Frequency Modulation (LFM) signal is more representative. It is used as an example to analyze the pulse compression process. The LFM signal can be expressed as:

$$
s(t)=A \exp \left(\mathrm{j} 2 \pi\left(f_{0} t+\frac{1}{2}\left(\frac{B}{T}\right) t^{2}\right)\right)
$$

In the formula, is the amplitude of the signal, is the initial frequency, is the signal bandwidth, and is the signal pulse width. The response of the corresponding matched filter can be expressed as:

$$
h(t)=s^{*}(-t)
$$

In the formula, is a complex conjugate signal with time inversion. The output results of the pulse compression can be expressed as

$$
p(t)=s(t) \otimes h(t)
$$

In the formula, means convolution. Combined with (1) (2) and the formula (3) can be expressed as:

\footnotetext{
* Corresponding author: gbgzbz@163.com
} 
(4)

$$
p(t)=A \sqrt{D} \operatorname{sinc}(\pi B t) \exp \left(\mathrm{j} 2 \pi f_{0} t\right)
$$

In the formula, $D$ is the pulse compression ratio, $D=B T$. From the formula (4), it can be seen that the result of pulse compression is a function. Compared with the original LFM signal, the target response pulse width is reduced by $D$ times and the amplitude has changed to $D$ times. It is obviously that the result of pulse compression has higher amplitude in target corresponding position, while the amplitude of other locations is much lower than the target location. This characteristic is used to measure the detection ability of signals.

\section{QUANTITATIVE ALGORITHM}

From the previous analysis results, it can be seen that the analysis often start with the peak value of the actual target. When analyzing the performance of integrated signal detection, it is generally considered whether the ratio between the peak value of the mainlobe and the side-lobe reaches the requirement. In the jamming effect evaluating, whether can the pulse compression results of the jamming signal effectively cover the response of the target position or form a higher false target response plays an important role in it. In order to realize evaluating the performance of the signal and the jamming effect automatically, a quantitative algorithm for this result is designed on the premise of not changing the information from the amplitude and it is designed based on the idea of $\mathrm{K}$ means clustering algorithm. The K-means algorithm was first proposed by Macqueen in 1967, and it is a simple unsupervised learning algorithm ${ }^{[8]}$. The algorithm owns the advantages of fast convergence and good clustering effect.

Considering that the analysis of the results of pulse compression is based on the peak of the image and the cluster result of the K-means algorithm is easily affected by the initial selection. In order to reduce these effects, the algorithm is improved properly and the quantization result of the algorithm is called the recognition degree. The improved algorithm is designed as follows:

Step1: If the amplitude of all points in pulse compression image is recorded as $A=\left\{a_{i}\right\}, i=1,2,3 \ldots$, and the target point is $a_{x}, x \leq i_{\max }$. For each point in the image, it will be selected when it satisfies $a_{i-1}<a_{i}<a_{i+1} \quad$. All the selected points are recorded as $b_{i}$, whose collection is written as $B$.
Step2: If $a_{x} \notin B$, that means the target point is not one of the selected points, the target point will not be confirmed as the true target point, and the recognition degree will be 0 ; If $\left\{a_{x}\right\}=B$, that means only the target point is the selected point, the target point will surely be confirmed as the true target point, and the recognition degree will be 1 . If these two conditions are not satisfied, the Step3 will be taken.

Step3: Cluster the selected points for analysis: Select 4 determined cluster initial centers as $p_{1}, p_{2}$, $p_{3}, p_{4} \cdot p_{1}$ represents the amplitude of the target point, that is $p_{1}=a_{x} ; p_{2}$ stands for the high sidelobe cluster center, and $p_{2}=a_{x} / 2 ; p_{3}=a_{x} / 31$ according to the ratio $30 \mathrm{~dB}$ of the main and sidelobes, which is set for the radar's clear recognition of target; As a low side-lobe clustering center, $p_{4}=0$ is taken. The result of cluster is recorded as $C_{1}, C_{2}, C_{3}, C_{4}$.

Step4: It is believed that the points of $C_{1}$ and $C_{2}$ have a strong influence on the detection of the target point. Therefore, all the points of $C_{1}$ and $C_{2}$ are taken as average values and recorded as $m_{1}$ and $m_{2}$; It is believed that the points of $C_{3}$ and $C_{4}$ have a little influence on the detection of the target point, so all points in $C_{3}$ and $C_{4}$ retain their maximum value, which are recorded as $m_{3}$ and $m_{4}$. Finally, the recognition degree is calculated according to the calculation method of geometric probability. If the recognition degree is recorded as $P$, it can be expressed as:

$$
P=\frac{a_{x}}{m 1+m 2+m 3+m 4}
$$

According to formula (5), it is obviously that if the recognition degree used for signal ability analysis, the higher the $P$ value, the better the signal pulse compression results; If used for the assessment of interference effects, the lower the $P$ value, the better the jamming effect. The algorithm process uses a flow chart as follows: 


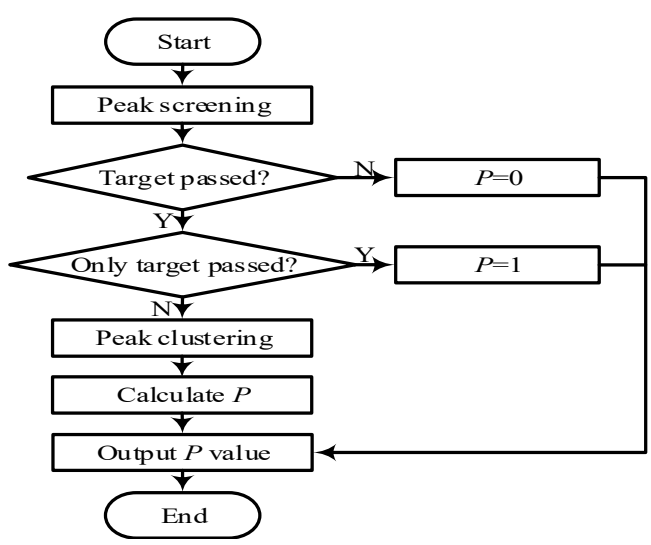

Fig.1, Algorithm flow chart

\section{SIMULATION}

It can be used that the same objects and simulation conditions, When assessing the signal detection performance, the LFM signal and the intrapulse modulated 20-symbol Continuous Phase ModulationLFM (CPM-LFM) signal were compared and analyzed in the absence of noise. When assessing the jamming effect, the jamming effects of radio frequency(RF) noise interference and convolution noise interference on the LFM signal under the same jamming-to-signal ratio are evaluated.

\subsection{Signal Performance Evaluation}

Simulation of LFM signal parameter is set to: Time width, bandwidth, sampling frequency, carrier frequency .Supplementary setting for CPM-LFM are association length, modulation coefficient and the random symbols are $[1,-1,-1,1,-1,1,1,-1,-1,1,1,-1,-1,1,-$ $1,1,1,-1,-1,1]$. Windowing technology is commonly used sidelobe suppression technology in pulse compression technology. For radar signals, windowing is often used to increase the ratio of main and sidelobes and improve signal capability. Therefore, the time-domain pulse compression simulations are performed on the above two signals, and the addition of a Hamming window is considered. Select the PLSR method and $\mathrm{K}$-means evaluation method to evaluate. The results are expressed as and, as follows:

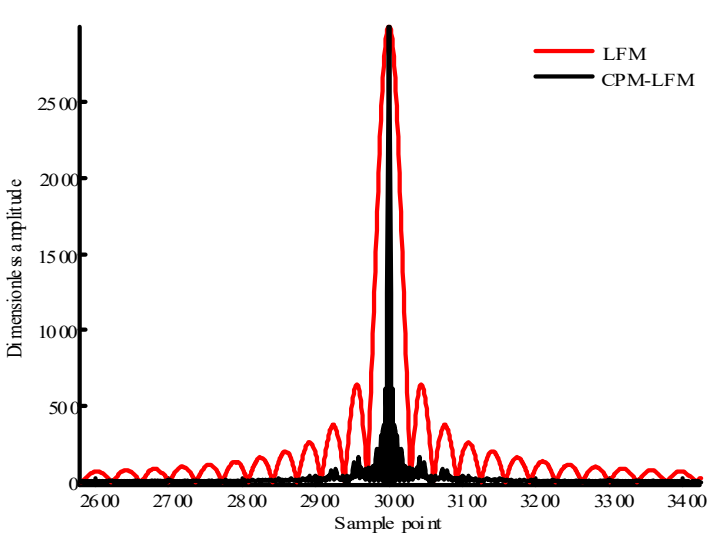

Fig.2, Pulse compression without Hamming window

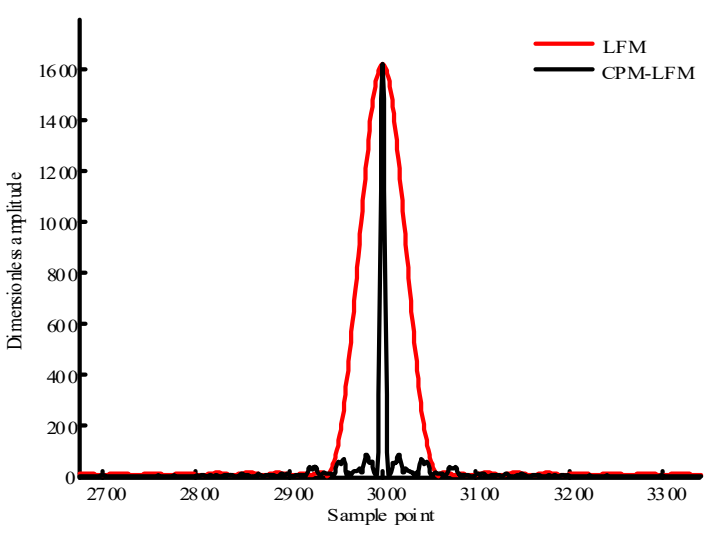

Fig.3, Pulse compression with Hamming window

The simulation results without windowing are: $S_{\mathrm{LFM}}=13.3987 \quad, \quad S_{\mathrm{CPM}-\mathrm{LFM}}=13.7019 \quad$, $P_{\mathrm{LFM}}=0.8136, P_{\mathrm{CPM}-\mathrm{LFM}}=0.8188 ;$ The simulation results of windowing are: $S_{\mathrm{LFM}}=40.6420$, $S_{\text {CPM-LFM }}=25.8898, P_{\text {LFM }}=0.9908, P_{\text {CPM-LFM }}=0.9404 \mathrm{w}$ hen using Hamming window. It can be seen from the results that the LFM signal has high sidelobes and its windowless recognition is slightly lower than that of the CPM-LFM signal. In other words, the pulse compression effect of the CPM-LFM signal is better than that of the LFM signal, which is consistent with the conclusion obtained by the PSLR method. The algorithm can also determine the performance of the signal pulse pressure results after windowing. Compared with the recognisability without window processing, the recognisability of the two signals after windowing has been significantly improved. It can also be concluded that the result of the two signals plus the Hamming window is that the LFM signal is superior to the CPM-LFM signal, and the conclusion is consistent. 


\subsection{Assessment of Jamming Effect}

Select the LFM signal as the target, taking the two interference technologies of radio frequency noise and convolution noise interference as examples, the proposed algorithm is used to analyze the interference effects of two interference technologies. The parameter is consistent with the parameter setting of the LFM signal in 3.1. Generally speaking, convolution noise interference as a form of smart interference, the radar jamming effect on the pulse compression system is superior to radio frequency noise interference under the same power conditions. Noise has a certain degree of randomness, it can cause fluctuations in the results. Therefore, 800 simulations are performed for each of the different ratios. The result obtained by the Monte Carlo method is used as the final identifiable degree under the condition of the jam to signal ratio. The simulation results are as follows:

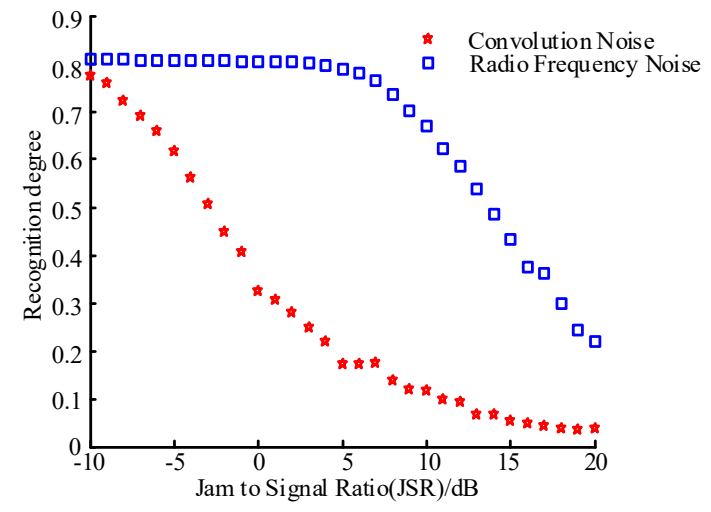

Fig.4, Recognition degree under different interference styles

It can be clearly seen from Figure 4 that the identifiable degree is basically the same under the influence of different interference patterns. The highest value approaches 0.8 , which is close to the previously no-noise identifiable degree; Recognition of the target point may be the convolution of the signal noise interference are set in the jam to signal ratio are lower than the range of variation of RF noise, it is superior to RF interference noise, as expected conclusion.

\section{CONCLUSIONS}

Aimed at the subjectivity existing in the result analysis of pulse compression image, a quantitative method based on clustering analysis is proposed. The simulation shows that the results of the algorithm are consistent with the traditional methods in the evaluation of signal pulse compression performance and jamming effect, and the correctness of the algorithm is demonstrated. The results can be applied to the evaluation system directly. However, the algorithm can not take into account the influence factors besides amplitude, such as the influence of main lobe width on target recognition. This is the place that still needs improving.

\section{References}

1. P.L.NIAN, C.H.LU. Jamming suppression of pulse compression radar based on frequency-shifting filter. Telecommunication Engineering, 57,1152 (2017).

2. Q.TONG. Evaluation of Jamming Effect of Pulsecompression Radar . XIDIAN University,47 (2014)

3. G.Q.SHEN, B.WU, B.HUANG. Research on radar anti-jamming performance based on spectrum sharing. China Radio, 5,35 (2011)

4. B.Y.WANG, R.B.GAN, J.R.ZHANG, Overview of Electronic Countermeasure Operational Effectiveness Evaluation.Electronic Information Warfare Technology, 32,54 (2017)

5. Y.C.WU. Research of active jamming to pulse compression radar. University of Electronic Science and Technology of China, 30 (2014)

6. H.LV. Rearch on jamming system of pulse compression radar and jamming effect evaluation, XIDIAN University,51 (2015).

7. S.W.ZHOU, J.YAN, Y.S.ZHOU. Study on squarewave frequency-shifting jamming to linear frequency modulation pulse compression radars.Journal of Telemetry, Tracking and Command,38,58 (2017)

8. J.C.HUANG. Improvement and Application of Kmeans algorithm .Changsha: Central South University, 5 (2013). 